\title{
A NON-CLUSTERING PROPERTY OF STATIONARY SEQUENCES ${ }^{1}$
}

\author{
By ARIF ZAMAN
}

\author{
The Florida State University
}

\begin{abstract}
For a random sequence of events, with indicator variables $X_{i}$, the behavior of the expectation $E\left\{\left(X_{k}+\ldots+X_{k+m-1}\right) /\left(X_{1}+\ldots+X_{n}\right)\right\}$ for $1 \leq k \leq k+$ $m-1 \leq n$ can be taken as a measure of clustering of the events. When the measure on the $X$ 's is i.i.d., or even exchangeable, a symmetry argument shows that the expectation can be no more than $m / n$. When the $X$ 's are constrained only to be a stationary sequence, the bound deteriorates, and depends on $k$ as well. When $m / n$ is small, the bound is roughly $2 m / n$ for $k$ near $n / 2$ and is like $(m / n) \log n$ for $k$ near 1 or $n$. The proof given is partly constructive, so these bounds are nearly achieved, even though there is room for improvement for other values of $k$.
\end{abstract}

1. Introduction. In considering portions of larger, but still finite strings of random variables, the following problem arose. If $X_{1}, \ldots, X_{n}$ is part of a stationary sequence of zeros and ones, one would not expect the ones within that portion to clump together, intuitively because each $X_{i}$ is as likely as any other to have the value one. Based on that intuitive argument, one could expect the expression $\sup _{P \in \mathscr{S}} E_{P}\left\{\left(X_{k}+\cdots+X_{k+m-1}\right) /\left(X_{1}+\cdots+X_{n}\right)\right\}$ (note: $0 / 0=0$ ) where $1 \leq k \leq k+m-1 \leq n$, and $\mathscr{S}$ is the set of stationary probability measures on binary sequences, to behave roughly like $m / n$. Indeed, if the probability $P$ is restricted to be i.i.d. or even exchangeable, a simple symmetry argument yields a supremum of $m / n$, achieved when the $X_{i}$ are identically 1 . For the case of stationarity, the upper bounds on the supremum for $m / n$ small are roughly $2 m / n$ when $k$ is near $n / 2$, and like $(m / n) \log n$ for $k$ closer to 1 or $n$ (Theorem 7). The key result is a constructive proof that finds the $P$ which achieves the supremum for the two cases of $m=1, k=1$, and $m=1, k=(n+1) / 2$ (Theorem 2).

I would like to thank Professor Michael Steele for insisting that this could be done, and Professor Larry Shepp for an improvement in the proof. I would also like to acknowledge the many simplifications and improvements suggested by the referee.

2. Results. We shall immediately narrow our concern to the simpler problem of finding bounds for

$$
R_{k, n}=\sup _{P \in \mathscr{S}} E_{P}\left\{\frac{X_{k}}{X_{1}+\cdots+X_{n}}\right\} \text { for } 1 \leq k \leq n .
$$

Received April 1982; revised May 1983.

${ }^{1}$ Partially supported by USARO grant DAAG-29-82-K-0168.

AMS 1980 subject classifications. Primary 60G10, 26D15.

Key words and phrases. Clustering, stationary sequences, cyclic sums. 
Notice that the variables $X_{n+1}, X_{n+2}, \cdots$ do not appear in the above expression, so only the marginal distribution of $\left(X_{1}, \ldots, X_{n}\right)$ affects the values of $R_{k, n}$. A small amount of notation is needed for the next theorem, which makes use of this observation.

A loop is a finite sequence $a_{1}, \cdots, a_{m}$ of zeros and ones. Subscripts out of range will be taken circularly, so that $a_{0}=a_{m}$, and $a_{m+1}=a_{1}$. For a loop $a$ and any positive integer $n$, the measure $P_{a, n}$ gives mass $1 / m$ to each of $\left(a_{1}, \cdots, a_{n}\right)$, $\left(a_{2}, \cdots, a_{n+1}\right), \cdots,\left(a_{m}, \cdots, a_{m+n-1}\right)$.

THEOREM 1. If a binary sequence $X$ has a stationary distribution, then the marginal distribution of $\left(X_{1}, \cdots, X_{n}\right)$ can be written as a convex combination of measures $P_{a, n}$ for $a \in A_{n}$, where $A_{n}$ is a finite set of loops. Moreover, every $P_{a, n}$ is the marginal of some infinite stationary distribution.

More details, and a proof of this can be found in Zaman (1983) or Hobby and Ylvasaker (1964). Since expectation is a linear functional, Theorem 1 allows replacing the maximization over $\mathscr{S}$ in equation 1 by maximization over $P_{a, n}$ for $a \in A_{n}$, yielding

$$
R_{k, n}=\max _{a \in A_{n}} E_{P_{a, n}}\left(X_{k} / \sum_{j=1}^{n} X_{j}\right) .
$$

Using the definition of $P_{a, n}$, the expectation can be further decomposed into

$$
E_{P_{a, n}}\left(\frac{X_{k}}{\sum_{j=1}^{n} X_{j}}\right)=\frac{1}{m} \sum_{i=1}^{m} \frac{a_{i+k}}{\sum_{j=1}^{n} a_{i+j}}
$$

where $m$ is the length of the loop $a$. In a completely unrelated problem, sums of the form given in the right side of equation 3 have been given the name cyclic sums, e.g. Daykin (1970).

Equations 2 and 3 convert the original probability problem of equation 1 into a finite maximization of a function over a set of loops. This maximization is performed for chosen values of $k$ in the appendix to prove the following key theorem.

THEOREM 2. (a) When $k=1$ or $n$, the maximum in equation 2 is achieved for $a=0^{n-1} 1^{\beta}$ (the notation $0^{n-1}$ refers to a block of $n-1$ zeros) for some number $\beta$ depending on $n$. (b) When $k=(n+1) / 2$ for odd $n$, the maximum in equation 2 is achieved for $a=0^{k-1} 1$.

Corollary 3. Define

$$
\alpha(n)=\sup _{\beta \geq 1}(n+\beta)^{-1} \sum_{i=1}^{\beta} 1 / i .
$$

Then,

$$
R_{k, n}=\left\{\begin{array}{lll}
\alpha(n-1) & \text { if } & k=1 \quad \text { or } n \\
2 /(n+1) & \text { if } & k=(n+1) / 2
\end{array}\right.
$$




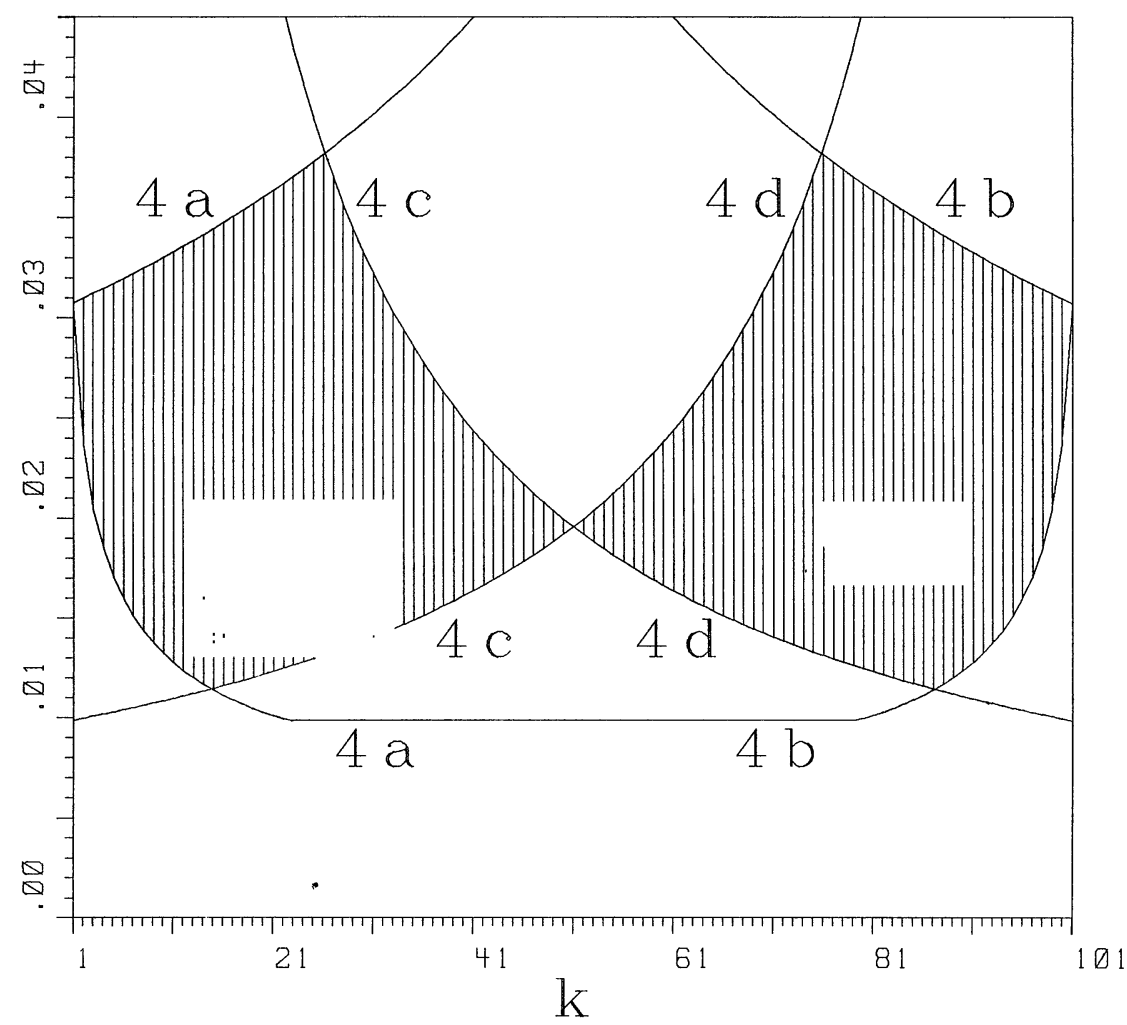

FIG. 1. Bounds on $R_{k, n}$ as a function of $k$, for $n=101$. The area between the upper and lower bounds of Theorem 4 is shaded to indicate the possible region for $R_{k, n}$. The different bounds are labeled by the equation number in Theorem 4.

The corollary is actually proved as a step in proving Theorem 2 , but can also be proved by writing out equation 3 for the loops given in Theorem 2 .

Using these equalities for $R_{1, n}$ and $R_{(n+1) / 2, n}$, a general bound for $R_{k, n}$ is easy to get. Theorems 4 and 5 do just that. The bounds of Theorem 4 are depicted graphically in Figure 1.

Theorem 4. Define

$$
\alpha(k, n)=\sup _{n-k \leq \beta}(k+\beta)^{-1}\left\{(n-k) / \beta+\sum_{i=n-k}^{\beta-1} 1 / i\right\} .
$$

Then
(a)
$\alpha(n-k, n) \leq R_{k, n} \leq \alpha(n-k)$ when $2 k-1 \leq n$
(b)
$\alpha(k-1, n) \leq R_{k, n} \leq \alpha(k-1)$ when $2 k-1 \geq n$
(c) $\quad 1 /(n+1-k) \leq R_{k, n} \leq 1 / k$
when $2 k-1 \leq n$
(d)
$1 / k \leq R_{k, n} \leq 1 /(n+1-k)$
when $2 k-1 \geq n$. 
Proof. Parts (b) and (d) follow from (a) and (c) respectively, once the symmetry condition

$$
R_{k, n}=R_{n-k+1, n}
$$

is established. To prove this, note that if $P_{a, n}$ is the distribution of $\left(X_{1}, \cdots, X_{n}\right)$ then the distribution of $\left(X_{n}, \cdots, X_{1}\right)$ is given by $P_{a^{\prime}, n}$ for $a^{\prime}=\left(a_{m}, \cdots, a_{1}\right)$. Now for any loop $a$,

$$
E_{P_{a, n}}\left(X_{k} / \sum_{j=1}^{n} X_{j}\right)=E_{P_{a^{\prime}, n}}\left(X_{n+1-k} / \sum_{j=1}^{n} X_{j}\right)
$$

from which equation 5 follows.

The upper bound in (a) follows from Corollary 3a by

$$
R_{k, n} \leq \sup _{P \in \mathscr{S}} E_{P}\left(X_{k} / \sum_{j=k}^{n} X_{j}\right)=R_{1, n+1-k}=\alpha(n-k) .
$$

Similarly, for part (c), the result of Corollary $3 \mathrm{~b}$ shows that for $2 k-1 \leq n$

$$
R_{k, n} \leq \sup _{P \in \mathscr{S}} E_{P}\left(X_{k} / \sum_{j=1}^{2 k-1} X_{j}\right)=R_{k, 2 k-1}=1 / k \text {. }
$$

The lower bounds have been included in the theorem to get some idea on the room for improvement of these bounds. It is conjectured that the actual values of $R_{k, n}$ are much closer to the lower bounds than to the upper bounds. The lower bound (a) is obtained by using equation 3 to get for $k \leq(n+1) / 2$

$$
\begin{aligned}
R_{k, n} & \geq \sup _{a=0^{n-k_{1} \beta}, k \leq \beta \leq n} E_{P_{a}}\left(X_{k} / \sum_{j=1}^{n} X_{j}\right) \\
& \geq \sup _{k \leq \beta \leq n}(n+\beta-k)^{-1}\left\{(k-1) / \beta+\sum_{i=k}^{\beta} 1 / i\right\} .
\end{aligned}
$$

The lower bound in (c) is achieved by letting $a=0^{n-k} 1$. For that value of $a$, if $2 k$ $-1 \leq n$ then by equation 3

$$
E_{P_{a}}\left(\frac{X_{k}}{\sum_{j=1}^{n} X_{j}}\right)=\frac{1}{n+1-k} .
$$

It is not difficult to find loops which give even higher lower bounds, but that does not seem to be the more fruitful direction of moving the bounds.

THEOREM 5.

$$
R_{k, n} \leq \frac{1+\log (n-1)}{n} \text { for } n \geq 3
$$

Before giving a proof, a logarithmic approximation for the function $\alpha$ will be established.

LEMMA 6.

$$
\frac{\log n-\log (\log n)-1}{n} \leq \alpha(n) \leq \frac{\log n}{n} \text { for } n \geq 3
$$


Proof. Let $\beta^{*}$ be a value of $\beta$ which achieves the maximum in equation 4 , so that

$$
\alpha(n)=\left(n+\beta^{*}\right)^{-1} \sum_{i=1}^{\beta^{*}} 1 / i .
$$

A crude bound to the harmonic series in equation 6 gives

$$
\alpha(n) \leq\left(1+\log \beta^{*}\right) /\left(n+\beta^{*}\right) .
$$

By calculus, the function $(1+\log x) /(n+x)$ for $x \geq 1$ reaches its maximum value of $\left(\log x^{*}\right) / n$ when $x^{*} \log x^{*}=n$. If $n>e, \log x^{*}$ can be bounded by

$$
\log n-\log \log n \leq \log x^{*} \leq \log n .
$$

Plugging this information about the maximum into equation 7

$$
\alpha(n) \leq\left(1+\log \beta^{*}\right) /\left(n+\beta^{*}\right) \leq\left(\log x^{*}\right) / n \leq(\log n) / n,
$$

establishing the second inequality of the lemma.

For the first inequality, let $x^{*}$ be as before, define $\beta=\left[x^{*}\right]$ (the integer part), and for notational convenience let $l=\log n-\log \log n$ which is the term on the left side of equation 8 . Then

$$
\begin{aligned}
\alpha(n) & \geq(n+\beta)^{-1} \sum_{i=1}^{\beta} 1 / i \geq\left(n+x^{*}\right)^{-1} \log x^{*} \\
& \geq(n+n / l)^{-1} l=n^{-1} l^{2} /(1+l)=n^{-1}\left\{l-1+(l+1)^{-1}\right\} \\
& >(l-1) / n .
\end{aligned}
$$

The last inequality substitutes a prettier expression at the cost of some precision. $\square$

The proof of Theorem 5 then amounts to the following. By equation 5

$$
\begin{aligned}
\max _{k} R_{k, n} & =\max _{k \leq(n+1) / 2} R_{k, n} \\
(\text { by Theorem 4a, c) } & \leq \max _{k \leq(n+1) / 2}\{(1 / k) \wedge \alpha(n-k)\} \\
(\text { by Lemma 6) } & \leq \max _{k \leq(n+1) / 2}\{(1 / k) \wedge \log (n-k) /(n-k)\} .
\end{aligned}
$$

Since $1 / k$ is decreasing and the second function increasing as $k$ increases, the maximum in equation 10 is attained at some $k=k^{*}$ for which the two functions are equal. Thus

$$
\max _{k} R_{k, n} \leq 1 / k^{*}=\log \left(n-k^{*}\right) /\left(n-k^{*}\right)=\left\{1+\log \left(n-k^{*}\right)\right\} / n,
$$

where the last expression follows by some algebra. Since $k^{*} \geq 1$, replacing it by 1 gives the claimed result in Theorem 5 .

Returning to the original problem as stated in the introduction, one can state the following theorem based only on the definition of $R_{k, n}$. 


\section{THEOREM 7.}

$$
\sup _{P \in \mathscr{S}} E_{P}\left\{\sum_{j=k}^{k+m-1} X_{j} / \sum_{j=1}^{n} X_{j}\right\} \leq \sum_{j=k}^{k+m-1} R_{j, n} .
$$

For example, this proves that for any stationary measure $P$,

$$
E_{P}\left\{\frac{X_{k}+\cdots+X_{k+m-1}}{X_{1}+\cdots+X_{n}}\right\} \leq \frac{m}{n}\{1+\log (n-1)\}
$$

and for blocks near the middle

$$
E_{P}\left\{\frac{X_{-k}+\cdots+X_{k}}{X_{-n}+\cdots+X_{n}}\right\} \leq \frac{1}{n+1}+2 \log \left(\frac{n}{n-k}\right) \leq \frac{2 k+1}{n-k}
$$

by using the values of $R_{k, n}$ given in Theorems 5 and $4 \mathrm{c}, \mathrm{d}$.

\section{APPENDIX}

Proof of Theorem 2a. The appendix will use equations 2, 3, 4, 5 and Lemma 6 from the previous section. It is to be noted that these do not use Theorem 2 in any way and are mainly definitional equations. To avoid repeating awkward summations, for the loop $a=a_{1}, \cdots, a_{m}$ we define

$$
\begin{aligned}
S(j, k) & =\sum_{i=j}^{k} a_{i}, \quad S_{i}=S(i-n+1, i), \\
T_{i} & =a_{i} / S_{i}, \quad T(j, k)=\sum_{i=j}^{k} T_{i} .
\end{aligned}
$$

By equation $5, R_{1, n}=R_{n, n}$. We will choose to work with $R_{n, n}$ for which equation 3 can be written as

$$
E_{P_{a, n}}\left(X_{n} / \sum_{j=1}^{n} X_{j}\right)=T(1, m) / m \text {. }
$$

Consider the case when $a$ is of the special form $0^{n-1} 1^{x}$ for some integer $x \leq n$. Working out the sums involved in equation A.1, for this $a$

$$
E_{P_{a, n}}\left(X_{n} / \sum_{j=1}^{n} X_{j}\right)=(n-1+x)^{-1} \sum_{i=1}^{x} 1 / i \leq \alpha(n-1) .
$$

It is easy to see that in equation A.2 equality is achieved for some value of $x \leq n$ which we shall denote by $\beta(n-1)$ (the argument $n-1$ will be assumed from now on). The proof that amongst the set of all loops, the given loop, $0^{n-1} 1^{\beta}$ maximizes the expectation will be done by contradiction. Assume there is some $a=a_{1}, \cdots, a_{m}$ and $\varepsilon>0$, for which

$$
T(1, m) / m>\alpha(n-1)+\varepsilon .
$$

The method of proof involves a stepwise modification of $a$. At each step the previous loop will be denoted by $a$, and the modified one by $a^{\prime}$. The variables $m^{\prime}$, for the length of $a^{\prime}$, as well as $S^{\prime}$ and $T^{\prime}$, will similarly be defined for $a^{\prime}$. After each step, for the modified sequence the inequality

$$
T^{\prime}\left(1, m^{\prime}\right) / m^{\prime}>\alpha(n-1)
$$

will be proved. Yet after a finite number of steps, the sequence $a^{\prime}$ will essentially look like $0^{n-1} 1^{\beta}$, providing the contradiction. 
Step 1. Let $m^{\prime}$ be a multiple of $m$, large enough so that $n / m^{\prime}<\varepsilon$, for the $\varepsilon$ in equation A.3, and also $m^{\prime}>5 n$ (this last restriction is not necessary, but allows the treatment of a loop as a long open string). We have $a=a_{1}, \cdots, a_{m}$. Let $a^{\prime}=0^{n-1} a_{n}, \cdots, a_{m^{\prime}}$.

To prove equation A.4 note that $a_{i}^{\prime} \leq a_{i}$, so $S_{i}^{\prime} \leq S_{i}$. So for $i=n, \cdots, m^{\prime}$ we have $T_{i}^{\prime} \geq T_{i}$, and for $i=1, \cdots, n-1, T_{i} \leq 1$. Hence

$$
T\left(1, m^{\prime}\right) \leq(n-1)+T^{\prime}\left(n, m^{\prime}\right) .
$$

Since $m^{\prime}$ is a multiple of $m$,

$$
\begin{aligned}
\alpha(n-1)+\varepsilon & <T(1, m) / m=T\left(1, m^{\prime}\right) / m^{\prime} \\
& \leq\left\{(n-1)+T^{\prime}\left(1, m^{\prime}\right)\right\} / m^{\prime} \leq \varepsilon+T^{\prime}\left(1, m^{\prime}\right) / m^{\prime}
\end{aligned}
$$

which proves equation A.4.

Step 2. Now $a=0^{n-1} a_{n}, a_{n+1}, \cdots, a_{m}$. Define $b=S(n, 2 n-1)$. Let $a^{\prime}=$ $0^{n-1} 1^{b} 0^{n-b} a_{2 n}, \cdots, a_{m}$.

Note that $a^{\prime}$ is simply $a$, with the block $a_{n}, \cdots, a_{2 n-1}$ rearranged so that all of its $b$ ones are to the left of its zeros. We pause to prove the following lemma about switching the order of a neighboring pair of 0 and 1 .

Lemma 8. Let $a$ and $a^{\prime}$ be two loops of the same length $m$, identical except that $a_{n+j}=a_{n+j+1}^{\prime}=0$ and $a_{n+j}^{\prime}=a_{n+j+1}=1$. If $a_{j+1}=0$, then

$$
T(1, m) \leq T^{\prime}(1, m) \text {. }
$$

Proof. The proof consists simply of noting that the only difference between $T_{i}$ and $T_{i}^{\prime}$ is $T_{2 n+j} \leq T_{2 n+j}^{\prime}, T_{n+j}=T_{n+j+1}^{\prime}$ and $T_{n+j+1}=T_{n+j}^{\prime}$.

Applying Lemma 8 repeatedly over a large block yields

CoROLlaRY 9. If a has a block of zeros $a_{j+1}=\ldots=a_{j+b}=0$ then construct $a^{\prime}$ by rearranging the block $a_{n+j}, \cdots, a_{n+j+b}$ so that the ones are to the left of the zeros, but otherwise $a$ and $a^{\prime}$ are identical. Then the conclusion of Lemma 8 is still valid.

Returning to Step 2 in the construction,

$$
\alpha(n-1)<T(1, m) / m \leq T^{\prime}\left(1, m^{\prime}\right) / m^{\prime},
$$

where the first inequality was established in Step 1, the second follows from Corollary 9.

Step 3. Now $a=0^{n-1} 1^{b} 0^{n-b} a_{2 n}, \cdots, a_{m}$. Let $a^{\prime}=0^{n-1} 1^{\beta} 0^{n-b} a_{2 n}, \cdots, a_{m}$ so that $m^{\prime}=m+\beta-b$.

By the definition of $\beta$ in equation A.2,

$$
\begin{aligned}
T(1, n+b-1) & =\sum_{i=1}^{b} 1 / i \leq(n+b-1) \alpha(n-1) \\
T^{\prime}(1, n+\beta-1) & =\sum_{i=1}^{\beta} 1 / i=(n+\beta-1) \alpha(n-1) .
\end{aligned}
$$


For the remaining values $i=n+b, \cdots, m$ we have $T_{i}=T_{i+\beta-b}^{\prime}$ if $\beta \geq b-1$. When $\beta<b-1$ the only difference is that $S_{i}>S_{i+\beta-b}^{\prime}$ for $i=2 n, \cdots, 2 n+b-$ $\beta-2$, so that in all cases

$$
T(n+b, m) \leq T^{\prime}\left(n+\beta, m^{\prime}\right) .
$$

Combining equations A.5 and A.6

$$
T(1, m)-T^{\prime}\left(1, m^{\prime}\right) \leq(b-\beta) \alpha(n-1) .
$$

This implies equation A.4 as can be seen by this simple lemma.

LEMMA 10. If $T(1, m)-T\left(1, m^{\prime}\right) \leq\left(m-m^{\prime}\right) \alpha$ and $T(1, m) / m>\alpha$ then $T^{\prime}\left(1, m^{\prime}\right) / m^{\prime}>\alpha$.

Proof. $\quad 0<T(1, m)-m \alpha<T^{\prime}\left(1, m^{\prime}\right)-m^{\prime} \alpha$. $\square$

Step 4. If $b>\beta$, return to Step 2; otherwise $n-b \geq n-\beta$, so the second block of zeros in $a$ has at least $n-\beta$ elements. Let $a_{c}$ be the first occurrence of a 1 in $a_{2 n+\beta-1}, \cdots, a_{m}$. Now $a=0^{n-1} 1^{\beta} 0^{n-\beta} a_{2 n}, \cdots, a_{c}, \cdots, a_{m}$. Let $a^{\prime}=0^{n-1} 1^{\beta} 0^{n-1} a_{c}$, $\cdots, a_{m}$, so that $m^{\prime}=m+2 n+\beta-c-1$.

Note that $T(1,2 n-1)=T^{\prime}(1,2 n+\beta-2), T_{2 n+\beta-1}^{\prime}=1$ and $T(c+1, m) \leq$ $T^{\prime}\left(2 n+\beta, m^{\prime}\right)$ so that

$$
T(1, m)-T^{\prime}\left(1, m^{\prime}\right) \leq T(2 n, 2 n+\beta-2)+T_{c}-1 .
$$

Let $d=S(2 n, 2 n+\beta-2)$ so that there are $n-d-1$ zeros in $a_{n}, \cdots, a_{2 n+\beta-2}$. Then each $S_{i}$ for $i=2 n, \cdots, 2 n+\beta-2$ sums at most $n-d-1$ zeros, and at least $d+1$ ones, i.e., each $S_{i} \geq d+1$. Since $a_{i}$ and hence $T_{i}$ is nonzero $d$ times for $i=2 n, \cdots, 2 n+\beta-2$

$$
T(2 n, 2 n+\beta-2) \leq d /(d+1) .
$$

We will separate out three cases, and in each case establish

$$
T(1, m)-T^{\prime}\left(1, m^{\prime}\right) \leq\left(m-m^{\prime}\right) \alpha(n-1),
$$

which would imply equation A.4 by Lemma 10 .

CASE 1. $2 n+\beta-1 \leq c<3 n$. Here $\left(m-m^{\prime}\right) \geq 0$ and $d=S_{c}-1$, so equations A.7 and A.8 imply

$$
T(1, m)-T^{\prime}\left(1, m^{\prime}\right) \leq\left(S_{c}+1\right) / S_{c}+1 / S_{c}-1=0,
$$

establishing equation A.9.

CASE 2. $c \geq 3 n$ and $n \neq 4,6,8$ or 10 .

Since $d \leq \beta-1$ and $m-m^{\prime} \geq n+1-\beta$, using equations A.7, A.8, we need to show

$$
(\beta-1) / \beta \leq(n+1-\beta) \alpha(n-1) .
$$




\begin{tabular}{rcr}
\multicolumn{3}{c}{ TABLE 1} \\
\hline $\boldsymbol{n}$ & $\beta(\boldsymbol{n}-\mathbf{1})$ & $\alpha(\boldsymbol{n}-\mathbf{1})$ \\
\hline 1 & 1 & 1.000000 \\
2 & 1 & .500000 \\
3 & 2 & .375000 \\
4 & 3 & .305556 \\
5 & 3 & .261905 \\
6 & 4 & .231481 \\
7 & 4 & .208333 \\
8 & 5 & .190278 \\
9 & 5 & .175641 \\
10 & 6 & .163333 \\
11 & 6 & .153125 \\
12 & 6 & .144118 \\
13 & 7 & .136466 \\
14 & 7 & .129643 \\
15 & 8 & .123539 \\
\hline
\end{tabular}

to prove equations A.9. Looking at Table 1, this holds for all given values of $n$ except 4, 6, 8, 10. For values beyond the table, equation A.7 was checked numerically up to $n=100$, and the logarithmic approximations of Lemma 6 will be used after that. Since $\beta$ maximizes equation A.2, we have

$$
\begin{aligned}
\alpha(n-1) & \geq\{n-1+(\beta-1)\}^{-1} \sum_{i=1}^{\beta-1} \frac{1}{i} \\
& =\left(\frac{n+\beta-1}{n+\beta-2}\right) \alpha(n-1)-\left(\frac{1}{n+\beta-2}\right)\left(\frac{1}{\beta}\right)
\end{aligned}
$$

which gives $\alpha(n-1) \leq 1 / \beta$. Since $\beta \alpha(n-1) \leq 1$ and $(\beta-1) / \beta \leq 1$,

$$
\begin{aligned}
(n+1-\beta) \alpha(n-1)-\frac{\beta-1}{\beta} & \\
& \geq(n+1) \alpha(n-1)-2 \\
\quad(\text { by Lemma } 6) & \geq(n+1) \frac{\log (n-1)-\log \log (n-1)-1}{n-1}-2 \\
& \geq 0 \text { for } n \geq 87 .
\end{aligned}
$$

The final inequality can be calculated for $n=87$, and since the penultimate expression is an increasing function of $n$, all larger $n$ must also satisfy it. But this establishes equation A.10 and hence A.9 for all $n \neq 4,6,8$, or 10 .

CASE 3. $c \geq 3 n$ and $n=4,6,8$, or 10 . This case is further broken into three subcases each involving a verification by Table 1 . 
(3a) If $c=3 n$ and $S_{c}>1$ then $T_{c} \leq 1 / 2$ so if

$$
(\beta-1) / \beta-1 / 2 \leq(n+1-\beta) \alpha(n-1)
$$

then equation A.9 is satisfied.

(3b) If $c=3 n$ and $S_{c}=1$ then $S(2 n+1,2 n+\beta-2)=0$, and so $T(2 n, 2 n+$ $\beta-2)=T_{2 n} \leq 1 / \beta$. Using this in equation A.7, we need to verify

$$
1 / \beta \leq(n+1-\beta) \alpha(n-1) \text {. }
$$

(3c) If $c>3 n$ then $m-m^{\prime} \geq n+2-\beta$ and we need

$$
(\beta-1) / \beta \leq(n+2-\beta) \alpha(n-1) .
$$

As these cases are exhaustive, and in each case equation A.4 is true, Step 4 is complete.

Step 5. Now $a=0^{n-1} 1^{\beta} 0^{n-1} a_{2 n+\beta-1}, \cdots, a_{m}$. Let $a^{\prime}=0^{n-1} a_{2 n+\beta-1}, \cdots, a_{m} 0^{n-1} 1^{\beta}$. Since $a^{\prime}$ is just a rotation of $a, T(1, m)=T^{\prime}\left(1, m^{\prime}\right)$, so equation A.4 will hold. Now, return to step 2 unless

$$
a=0^{n-1} 1^{\beta} 0^{n-1} 1^{\beta} \ldots 0^{n-1} 1^{\beta} .
$$

At every return to Step 2, some elements of the original sequence are deleted or reordered into blocks of $0^{n-1} 1^{\beta}$. Since no new disordered elements are created at any step, the procedure must stop after a finite number of steps. Since at each step equation A.4 was verified, for the final $a$ of equation A.11 we must have

$$
T(1, m) / m>\alpha(n-1)
$$

yet simply computing,

$$
T(1, m) / m=(n-1+\beta)^{-1} \sum_{i=1}^{\beta} 1 / i=\alpha(n-1)
$$

providing the contradiction which proves the theorem. $\square$

Proof of Theorem 2b. Let $n$ be odd, $k=(n+1) / 2$, and $a=a_{1}, \cdots, a_{m}$. As notation, define

$$
S\left(j, j^{\prime}\right)=\sum_{i=j}^{j^{\prime}} a_{i}, \quad T_{i}=\frac{a_{i+k}}{S(i+1, i+n)}, \quad T\left(j, j^{\prime}\right)=\sum_{i=j}^{j^{\prime}} T_{i}
$$

so that equation 3 can be written as

$$
E_{P_{a, n}}\left(X_{k} / \sum_{j=1}^{n} X_{j}\right)=T(1, m) / m
$$

For any loop $a$,

$$
\begin{aligned}
T(1, k) & =\sum_{i=1}^{k} a_{i+k} / S(i+1, i+n) \\
& \leq \sum_{i=1}^{k} a_{i+k} / S(k+1, n+1)=1 .
\end{aligned}
$$

As this holds for all loops, it will also hold for the loop $\left(a_{h k+1}, a_{h k+2}, \cdots, a_{h k+n}\right)$ for any integer $h$. Thus

$$
T(h k+1,(h+1) k) \leq 1 \text { for } h=0,1,, \cdots .
$$


Adding these up for $h=0,1, \cdots, m-1$,

(A12) $\quad m>\sum_{h=0}^{m-1} T((h k+1),(h+1) k)=T(1, m k)=k T(1, m)$,

because $a$ is periodic with period $m$. Rewriting A.12 gives

$$
T(1, m) / m \leq 1 / k=2 /(n+1)
$$

for any loop $a$. On the other hand, it is straightforward to verify that the loop $a=0^{k-1} 1$ achieves the upper bound in equation A.13, thus proving Theorem $2 \mathrm{~b}$ and Corollary $3 \mathrm{~b}$ simultaneously.

\section{REFERENCES}

Daykin, D. E. (1970). Inequalities for certain cyclic sums. Proc. Edin. Math. Soc. (Ser. 2) 17 257262.

HoBBY, C. and YLVASAKER, D. (1964). Some structure theorems for stationary probability measures on finite state sequences. Ann. Math. Statist. 35 550-556.

Zaman, A. (1983). Stationarity on finite strings and shift register sequences. Ann. Probab. 11 678684.

DEPARTMENT OF STATISTICS Florida STATE UNIVERSITY

TAllahassee, FloRIDA 32306 